\title{
COTYPE AND ABSOLUTELY SUMMing LINEAR OPERATORS
}

\author{
Geraldo Botelho, Daniel Pellegrino* and Pilar Rueda ${ }^{\dagger}$
}

\begin{abstract}
Cotype is used in this paper to prove new results concerning the existence of approximable (hence compact) non-absolutely summing linear operators between Banach spaces. We derive consequences that extend/generalize/complement some classical results of Bennett [1, Davis and Johnson [5], and Lindenstrauss and Pełczyński [7]. We also point out that some of our results are sharp.
\end{abstract}

\section{Introduction}

The central role played by the concept of cotype in the theory of absolutely summing linear (and nonlinear) operators is well known (the linear case is folklore, for the nonlinear case, see, e.g., [2, 9]). Cotype has been traditionally used to show that operators between certain spaces are always absolutely summing, that is, cotype has been a valuable tool to show how abundant absolutely summing operators are. In this paper we show how cotype can also be used to prove the existence of non-absolutely summing operators. For instance we prove in Corollary 2.6 that if $X$ and $Y$ are infinite-dimensional Banach spaces and $\cot Y>\max \{2, r\}$, then there exists an approximable (hence compact, weakly compact, completely continuous, strictly singular, strictly cosingular, etc) linear operator from $X$ to $Y$ that fails to be $r$-summing. We also show that some of our results are somehow sharp.

These results and their consequences follow the line of classical results of Bennett [1], Davis and Johnson [5], Lindenstrauss and Pełczyński [7] and complement/generalize some of their information. More precisely:

- Non-coincidence results for absolutely summing operators between $\ell_{p}$-spaces of [1] are shown to hold true for operators between much more general spaces (Proposition [2.9);

\footnotetext{
*Supported by CNPq Grant 308084/2006-3.

†Supported by Ministerio de Ciencia e Innovación MTM2008-03211/MTM. 2000 Mathematics Subject Classification: 46B20, 47B10.
} 
- The existence of compact non-absolutely summing operators on superreflexive spaces of [5] is extended to operators on arbitrary infinite-dimensional spaces provided that the infimum of the cotypes of the range spaces is not 2 (Corollary 2.6);

- In [7] it is proved that if every operator from $X$ to $Y$ is absolutely summing, then $\cot X=2$; in Proposition 2.8(c) we improve this result by showing that with weaker conditions we can conclude that $\cot X=\cot Y=2$.

If every continuous linear operator from $X$ to any Banach space $Y$ is $(q, 1)$ summing, we can call on a deep result due to Maurey and Pisier [8] (cf. [6, Theorem 14.5]) to conclude that $\cot X \leq q$. In Corollary 2.3 we combine Maurey and Pisier's result with Theorem 2.1 to show that the conclusion $\cot X \leq q$ can be derived from the weaker condition that every approximable linear operator from $X$ to some infinite-dimensional Banach space $Y$ with no finite cotype is $(q, 1)$-summing.

\section{Notation and background}

Throughout this paper $X$ and $Y$ will stand for Banach spaces over $\mathbb{K}=\mathbb{R}$ or $\mathbb{C}$ and $\mathcal{L}(X, Y)$ means the space of bounded linear operators from $X$ to $Y$ endowed with the usual sup norm. The closed unit ball of $X$ is denoted by $B_{X}$ and the topological dual of $X$ by $X^{*}$. We follow the standard terminology of absolutely summing operators (see [6]). Given $x_{1}, \ldots, x_{n} \in X$,

$$
\left\|\left(x_{j}\right)_{j=1}^{n}\right\|_{w, p}=\sup _{\varphi \in B_{X^{*}}}\left(\sum_{j=1}^{n}\left|\varphi\left(x_{j}\right)\right|^{p}\right)^{\frac{1}{p}} .
$$

A linear operator $u \in \mathcal{L}(X, Y)$ is absolutely $(q, p)$-summing if there is a constant $C \geq 0$ such that

$$
\left(\sum_{j=1}^{n}\left\|u\left(x_{j}\right)\right\|^{q}\right)^{\frac{1}{q}} \leq C\left\|\left(x_{j}\right)_{j=1}^{n}\right\|_{w, p}
$$

for every $n \in \mathbb{N}$ and $x_{1}, \ldots, x_{n} \in X$. The space of absolutely $(q, p)$-summing linear operators from $X$ to $Y$ is denoted by $\Pi_{q, p}(X, Y)$. The $(q, p)$-summing norm of $u$, defined as the infimum of the constants $C$ working in the inequality, is represented by $\pi_{q, p}(u)$. If $p=q$ we say simply absolutely $p$-summing operator and write $\Pi_{p}(X, Y)$ and $\pi_{p}(u)$ for the space of absolutely $p$-summing operators and the $p$-summing norm of $u$, respectively.

The notation $\cot X$ denotes the infimum of the cotypes assumed by $X$. The identity operator on $X$ is denoted by $i d_{X}$.

By $\mathcal{A}(X, Y)$ we mean the subspace of $\mathcal{L}(X, Y)$ of approximable operators, that is, the closure of the finite rank operators in the usual operator sup-norm. 


\section{Results}

Our first aim is to prove that if $\cot Y>\max \{2, r\}$ and $X$ is infinite-dimensional, then one can find an approximable non-r-summing linear operator from $X$ to $Y$. Consequences of this result complement and generalize classical results from [1, 5, 7]. Furthermore, it is sharp in the sense that it is not valid for operators into cotype 2 spaces.

The proof of the next result is a far reaching refinement of the arguments used in [9, 10] for spaces with unconditional Schauder basis, which, in their turn, were inspired in the classical paper [7].

Theorem 2.1. Let $X$ and $Y$ be infinite-dimensional Banach spaces, $2 \leq p<$ $\infty$ and $q, r>0$ such that $\cot Y \geq p>q \geq r$. If $\mathcal{A}(X, Y) \subseteq \Pi_{q, r}(X, Y)$, then $i d_{X}$ is $\left(\frac{p q}{p-q}, r\right)$-summing.

Proof. Since $Y$ is infinite-dimensional, from [6, Theorem 14.5] we have that $\cot Y=\sup \left\{2 \leq s \leq \infty ; Y\right.$ finitely factors the formal inclusion $\left.\ell_{s} \hookrightarrow \ell_{\infty}\right\}$, and from [6, p. 304] we know that this supremum is attained. So $Y$ finitely factors the formal inclusion $\ell_{p} \hookrightarrow \ell_{\infty}$, that is, there exist $C_{1}, C_{2}>0$ such that for every $n \in \mathbb{N}$, there are $y_{1}, \ldots, y_{n} \in Y$ so that

$$
C_{1}\left\|\left(a_{j}\right)_{j=1}^{n}\right\|_{\infty} \leq\left\|\sum_{j=1}^{n} a_{j} y_{j}\right\| \leq C_{2}\left(\sum_{j=1}^{n}\left|a_{j}\right|^{p}\right)^{\frac{1}{p}}
$$

for every $a_{1}, \ldots, a_{n} \in \mathbb{K}$.

From the inequality $\|\cdot\| \leq \pi_{q, r}(\cdot)$ and the assumption $\mathcal{A}(X, Y) \subseteq \Pi_{q, r}(X, Y)$ it follows easily that $\mathcal{A}(X, Y)$ is closed with respect to $\pi_{q, r}$. So we can use the Open Mapping Theorem to get a constant $K>0$ such that $\pi_{q, r}(u) \leq K\|u\|$ for all linear operators $u \in \mathcal{A}(X, Y)$. Let $n \in \mathbb{N}$ and $x_{1}, \ldots, x_{n} \in X$ be given. Consider $x_{1}^{*}, \ldots, x_{n}^{*} \in B_{X^{*}}$ so that $x_{j}^{*}\left(x_{j}\right)=\left\|x_{j}\right\|$ for every $j=1, \ldots, n$. Let $\mu_{1}, \ldots, \mu_{n}$ be scalars such that $\sum_{j=1}^{n}\left|\mu_{j}\right|^{s}=1$, where $s=\frac{p}{q}$. Let

$$
u: X \longrightarrow Y, u(x)=\sum_{j=1}^{n}\left|\mu_{j}\right|^{\frac{1}{q}} x_{j}^{*}(x) y_{j} .
$$

It is clear that $u$ is a finite rank operator, so $u \in \mathcal{A}(X, Y)$. Moreover, for every $x \in X$,

$$
\begin{aligned}
\|u(x)\| & =\left\|\sum_{j=1}^{n}\left|\mu_{j}\right|^{\frac{1}{q}} x_{j}^{*}(x) y_{j}\right\| \leq C_{2}\left(\left.\left.\sum_{j=1}^{n}|| \mu_{j}\right|^{\frac{1}{q}} x_{j}^{*}(x)\right|^{p}\right)^{\frac{1}{p}} \\
& \leq C_{2}\left(\sum_{j=1}^{n}\left|\mu_{j}\right|^{\frac{p}{q}}\right)^{\frac{1}{p}}\|x\|=C_{2}\|x\| .
\end{aligned}
$$


We thus have $\pi_{q, r}(u) \leq K\|u\| \leq K C_{2}:=K_{1}$. Note that for $k=1, \ldots, n$, we have

$$
\begin{aligned}
\left\|u\left(x_{k}\right)\right\| & =\left\|\sum_{j=1}^{n}\left|\mu_{j}\right|^{\frac{1}{q}} x_{j}^{*}\left(x_{k}\right) y_{j}\right\| \geq C_{1}\left\|\left(\left|\mu_{j}\right|^{\frac{1}{q}} x_{j}^{*}\left(x_{k}\right)\right)_{j=1}^{n}\right\|_{\infty} \\
& \geq C_{1}\left|\mu_{k}\right|^{\frac{1}{q}} x_{k}^{*}\left(x_{k}\right)=C_{1}\left|\mu_{k}\right|^{\frac{1}{q}}\left\|x_{k}\right\| .
\end{aligned}
$$

So we have

$$
\begin{aligned}
\left(\sum_{j=1}^{n}\left\|x_{j}\right\|^{q}\left|\mu_{j}\right|\right)^{\frac{1}{q}} & =\left(\sum_{j=1}^{n}\left(\left\|x_{j}\right\|\left|\mu_{j}\right|^{\frac{1}{q}}\right)^{q}\right)^{\frac{1}{q}} \\
& \leq C_{1}^{-1}\left(\sum_{j=1}^{n}\left\|u\left(x_{j}\right)\right\|^{q}\right)^{\frac{1}{q}} \\
& \leq C_{1}^{-1} \pi_{q, r}(u)\left\|\left(x_{j}\right)_{j=1}^{n}\right\|_{w, r} .
\end{aligned}
$$

Observing that this last inequality holds whenever $\sum_{j=1}^{n}\left|\mu_{j}\right|^{s}=1$ and that $\frac{1}{s}+$ $\frac{1}{\frac{s}{s-1}}=1$, we obtain

$$
\begin{aligned}
\left(\sum_{j=1}^{n}\left\|x_{j}\right\|^{\frac{s}{s-1} q}\right)^{\frac{1}{s-1}} & =\sup \left\{\left|\sum_{j=1}^{n} \mu_{j}\left\|x_{j}\right\|^{q}\right| ; \sum_{j=1}^{n}\left|\mu_{j}\right|^{s}=1\right\} \\
& \leq \sup \left\{\sum_{j=1}^{n}\left|\mu_{j}\right|\left\|x_{j}\right\|^{q} ; \sum_{j=1}^{n}\left|\mu_{j}\right|^{s}=1\right\} \\
& \leq\left(C_{1}^{-1} \pi_{q, r}(u)\left\|\left(x_{j}\right)_{j=1}^{n}\right\|_{w, r}\right)^{q} \\
& \leq\left(C_{1}^{-1} \cdot K_{1} \cdot\left\|\left(x_{j}\right)_{j=1}^{n}\right\|_{w, r}\right)^{q},
\end{aligned}
$$

and then

$$
\left(\sum_{j=1}^{n}\left\|x_{j}\right\|^{\frac{s}{s-1} q}\right)^{\frac{1}{s-1} q} \leq C_{1}^{-1} \cdot K_{1} \cdot\left\|\left(x_{j}\right)_{j=1}^{n}\right\|_{w, r} .
$$

Since $\frac{s}{s-1} q=\frac{p q}{p-q}, n$ and $x_{1}, \ldots, x_{n} \in X$ are arbitrary, we conclude that $i d_{X}$ is $\left(\frac{p q}{p-q}, r\right)$-summing.

Corollary 2.2. Let $X$ and $Y$ be infinite-dimensional Banach spaces, $2 \leq p<$ $\infty$ and $q>1$ such that $\cot Y \geq p>q$. If $\frac{p q}{p-q}>2$ and $\mathcal{A}(X, Y) \subseteq \Pi_{q, 1}(X, Y)$, then $X$ has cotype $\frac{p q}{p-q}$.

Proof. From Theorem 2.1 we conclude that $i d_{X}$ is $\left(\frac{p q}{p-q}, 1\right)$-summing. Since $\frac{p q}{p-q}>2$, using a result due to Talagrand (see [12]) we conclude that $X$ has cotype $\frac{p q}{p-q}$. 
Observe that the result above is an interesting improvement of the linear case of [9, Corollary 2], because there, contrary to here, a Schauder basis for $X$ is required.

A well known result due to Maurey and Pisier asserts that $\cot X=\inf \{2 \leq$ $\left.q \leq \infty: i d_{X} \in \Pi_{q, 1}(X, X)\right\}$ ([6, Theorem 14.5] and [8]). Next corollary is a significant improvement of the linear cases of [9, Theorem 7] and [4, Corollary 2.5] and complements information from [8]:

Corollary 2.3. Let $X$ be an infinite-dimensional Banach space. If there is an infinite-dimensional Banach space $Y$ with no finite cotype and such that $\mathcal{A}(X, Y) \subseteq \Pi_{q, 1}(X, Y)$, then $\cot X \leq q$.

Proof. The assumption $\cot Y=\infty$ allows us to make $p \longrightarrow \infty$ in Theorem 2.1 to conclude that $i d_{X}$ is $(q+\varepsilon, 1)$-summing for every $\varepsilon>0$. Hence $q \geq 2$ by $[6$, Theorem 10.5] and $\cot X \leq q$ by [6, Theorem 14.5].

Remark 2.4. Let $X$ be an infinite-dimensional Banach space with an unconditional Schauder basis $\left(x_{n}\right)$. Define

$$
\mu_{\left(x_{n}\right)}=\inf \left\{t:\left(a_{j}\right) \in \ell_{t} \text { whenever } \sum_{j=1}^{\infty} a_{j} x_{j} \in X\right\} .
$$

In [9, Theorem 7] it is shown that if $Y$ has no finite cotype and $\Pi_{q, 1}(X, Y)=$ $\mathcal{L}(X, Y)$, then $\mu_{\left(x_{n}\right)} \leq q$. Corollary 2.3 improves this information in three directions: (i) a Schauder basis is not needed in Corollary 2.3, (ii) if $X$ has an unconditional Schauder basis, the conclusion $\cot X \leq q$ of Corollary 2.3 is stronger than the conclusion $\mu_{\left(x_{n}\right)} \leq q$ of [9, Theorem 7], (iii) it is enough to have $\mathcal{A}(X, Y) \subseteq \Pi_{q, 1}(X, Y)$ instead of $\Pi_{q, 1}(X, Y)=\mathcal{L}(X, Y)$.

Now we are in the position to prove our main results.

Theorem 2.5. Let $X$ and $Y$ be infinite-dimensional Banach spaces. If $\cot Y \geq$ $p>q \geq r>\frac{2 p q}{p q+2 p-2 q}$, then there exists an approximable non- $(q, r)$-summing linear operator from $X$ to $Y$.

Proof. Assume that $\mathcal{A}(X, Y) \subseteq \Pi_{q, r}(X, Y)$. By Theorem 2.1 we have that $i d_{X}$ is $\left(\frac{p q}{p-q}, r\right)$-summing. Since $i d_{X} \neq 0$ we have $r \leq \frac{p q}{p-q}$. By [6, Theorem 10.5] it follows that $\frac{1}{r}-\frac{p-q}{p q} \geq \frac{1}{2}$, that is $r \leq \frac{2 p q}{p q+2 p-2 q}$.

A classical result due to Davis and Johnson [5] asserts that if $X$ is superreflexive, then there exists a compact non- $r$-summing linear operator from $X$ to any infinite-dimensional space $Y$. Let us see that for operators with range spaces $Y$ with $\cot Y>\max \{2, r\}$ there is no need to impose any condition on the domain space $X$ :

Corollary 2.6. Let $X$ and $Y$ be infinite-dimensional Banach spaces with $\cot Y>\max \{2, r\}$. Then there exists an approximable (hence compact) non$r$-summing linear operator from $X$ to $Y$. 
Proof. For $2 \leq r<\cot Y$ the result follows from Theorem 2.5, with $p=\cot Y$ and $q=r$, because $\frac{2 p q}{p q+2 p-2 q}<2$. The case $r<2$ is a consequence of the inclusion theorem [6, Theorem 2.8].

Remark 2.7. (a) Grothendieck's theorem $\Pi_{1}\left(\ell_{1}, \ell_{2}\right)=\mathcal{L}\left(\ell_{1}, \ell_{2}\right)$ makes clear that Theorem 2.5 and Corollary 2.6 are sharp in the sense that they are not valid for operators with range cotype 2 spaces. The case $\cot Y>2$ is also close to optimality. In fact, from [6, Corollary 10.10] we know that if $Y$ is an $\mathcal{L}_{q}$-space $(q>2)$ and $r>q=\cot Y$, then

$$
\Pi_{r}\left(c_{0} ; Y\right)=\mathcal{L}\left(c_{0} ; Y\right) .
$$

(b) It is worth mentioning that $\mathcal{A}$ is contained in any closed operator ideal $\mathcal{I}$ (e.g., operators that are compact, weakly, compact, completely continuous, strictly singular, strictly cosingular, Banach-Saks property, Rosenthal property, etc - see [11]). So, for any closed operator ideal $\mathcal{I}$, if $X$ and $Y$ are infinite-dimensional Banach spaces with $\cot Y>\max \{2, r\}$, then there exists a non- $r$-summing linear operator from $X$ to $Y$ belonging to $\mathcal{I}$.

A classical theorem due to Lindenstrauss and Pełczyński [7, Proposition 8.1(2)] asserts that if $X$ and $Y$ are infinite-dimensional and $\Pi_{1}(X, Y)=\mathcal{L}(X, Y)$, then $\cot X=2$. Part (c) of the next proposition improves this result in the sense that a stronger conclusion is obtained from a weaker assumption.

\section{Proposition 2.8.}

(a) Let $X$ be an infinite-dimensional Banach space. If there is an infinitedimensional Banach space $Y$ with finite cotype such that $\mathcal{A}(X, Y) \subseteq \Pi_{\frac{2 \cot Y}{2+\cot Y}, 1}(X, Y)$, then $X$ has the Orlicz property (that is, $i d_{X}$ is $(2,1)$-summing).

(b) If $X$ is infinite-dimensional and $\mathcal{A}(X, Y) \subseteq \Pi_{r}(X, Y)$ for some infinitedimensional Banach space $Y$ and some $1 \leq r<2$, then $\cot Y=2$.

(c) If $X$ and $Y$ are infinite-dimensional Banach spaces and every approximable linear operator from $X$ to $Y$ is 1-summing, then $\cot X=\cot Y=2$.

Proof. (a) Choose $r=1, p=\cot Y$ and $q=\frac{2 p}{2+p}$ in Theorem 2.1.

(b) This is immediate from Theorem 2.5. In fact, if $\cot Y>2$, we can take $p=$ $\cot Y$ and $q=r$ in Theorem 2.5 and conclude that $\mathcal{A}(X ; Y)$ is not contained in $\Pi_{r}(X ; Y)$.

(c) Putting $r=1$ in (b) we conclude that $\cot Y=2$, then (a) yields that $i d_{X}$ is $(2,1)$-summing. Therefore $\cot X=2$ by [6, Theorem 14.5].

As announced in the introduction, we shall improve substantially the following information from Bennett [1, Proposition 5.2(i)]:

- $\Pi_{q, 1}\left(\ell_{1}, \ell_{p}\right) \neq \mathcal{L}\left(\ell_{1}, \ell_{p}\right)$ whenever $2 \leq p<\infty$ and $q<\frac{2 p}{2+p}$

- $\Pi_{q, 2}\left(\ell_{1}, \ell_{p}\right) \neq \mathcal{L}\left(\ell_{1}, \ell_{p}\right)$ whenever $2 \leq p<\infty$ and $q<p$.

- $\Pi_{q, 1}\left(\ell_{1} ; \ell_{\infty}\right) \neq \mathcal{L}\left(\ell_{1} ; \ell_{\infty}\right)$ whenever $1 \leq q<2$.

Our improvement says that $\ell_{1}$ may be replaced by any infinite-dimensional 
Banach space, $\ell_{p}$ may be replaced by any infinite-dimensional Banach space with $\cot Y=p$ and the existence of a non-absolutely summing operator can be replaced by the existence of an approximable non-absolutely summing operator:

Proposition 2.9. Let $X$ and $Y$ be infinite-dimensional Banach spaces.

(a) If $\cot Y<\infty$, then there exists an approximable non- $(q, 1)$-summing linear operator from $X$ to $Y$ for every $q<\frac{2 \cot Y}{2+\cot Y}$.

(b) If $\cot Y<\infty$, then there exists an approximable non- $(q, 2)$-summing linear operator from $X$ to $Y$ for every $q<\cot Y$.

(c) If $\cot Y=\infty$, then there exists an approximable non-( $q, 1)$-summing linear operator from $X$ to $Y$ for every $1 \leq q<2$.

Proof. (a) Make $r=1$ in Theorem 2.5 and observe that $q<\frac{2 p}{2+p} \Longleftrightarrow 1>$ $\frac{2 p q}{p q+2 p-2 q}$.

(b) Just make $r=2$ in Theorem 2.5.

(c) Otherwise, from Corollary 2.3 it would follow that $\cot X \leq q<2$.

Remark 2.10. Proposition 2.9 is sharp: for example, from [1, Proposition $5.2(\mathrm{i})]$ we know that $\Pi_{\frac{2 p}{2+p}, 1}\left(\ell_{1}, \ell_{p}\right)=\mathcal{L}\left(\ell_{1}, \ell_{p}\right)$ for $p \geq 2$ and $\Pi_{p, 2}\left(\ell_{1}, \ell_{p}\right)=$ $\mathcal{L}\left(\ell_{1}, \ell_{p}\right)$ for $p \geq 2$.

A final application of our results illustrates, in one single result, the well known relevance of the space $\ell_{1}$ and of the concept of cotype in the theory of absolutely summing operators:

Proposition 2.11. Let $X$ and $Y$ be infinite-dimensional Banach spaces. Let $2 \leq r<\cot Y$ and $q \geq r$ be such that $\Pi_{q, r}(X, Y)=\mathcal{L}(X, Y)$. Then $\mathcal{L}\left(\ell_{1}, \ell_{\cot Y}\right)=$ $\Pi_{q, r}\left(\ell_{1}, \ell_{\cot Y}\right)$.

Proof. Combine Theorem 2.5 with [1, Proposition 5.2(iv)].

Final remark. The results of this paper can be adapted, mutatis mutandis, to absolutely summing homogeneous polynomials and multilinear mappings. For homogeneous polynomials, Theorem 2.1 reads as: if $2 \leq p<\infty$ and $q>0$ are such that $\cot Y \geq p>q \geq \frac{r}{m}$, and the the space of absolutely $(q, r)$-summing $m$-homogeneous polynomials from $X$ to $Y$ contains the closure (in the usual sup-norm) of the space of all continuous $m$-homogeneous polynomials of finite type, then $i d_{X}$ is $\left(\frac{m p q}{p-q}, r\right)$-summing. The resulting consequences and related results follow accordingly.

\section{References}

[1] G. Bennett, Schur multipliers, Duke Math. Journal 44 (1977), 603-639. 
[2] G. Botelho, Cotype and absolutely summing multilinear mappings and homogeneous polynomials, Proc. Roy. Irish Acad Sect. A 97 (1997), 145-153.

[3] G. Botelho and D. Pellegrino, Absolutely summing polynomials on Banach spaces with unconditional Schauder basis, J. Math. Anal. Appl. 321 (2006), $50-58$.

[4] G. Botelho and D. Pellegrino, Absolutely summing linear operators into spaces with no finite cotype, to appear in Bull. Belg. Math. Soc. Simon Stevin.

[5] W. J. Davis and W. B. Johnson, Compact non-nuclear operators, Studia Math. 51 (1974), 81-85.

[6] J. Diestel, H. Jarchow, A. Tonge, Absolutely summing operators, Cambridge University Press, 1995.

[7] J. Lindenstrauss and A. Pełczyński, Absolutely summing operators in $\mathcal{L}_{p}$ spaces and their applications. Studia Math 29 (1968), 275-326.

[8] B. Maurey and G. Pisier Séries de variables aléatoires vectorielles indépendantes et propriétés géométriques des espaces de Banach, Studia Math. 58 (1976), 45-90.

[9] D. Pellegrino, Cotype and absolutely summing homogeneous polynomials in $\mathcal{L}_{p}$ spaces, Studia Math. 157 (2003), 121-131.

[10] D. Pellegrino, On scalar-valued nonlinear absolutely summing mappings, Ann. Polon. Math. 83 (2004), 281-288.

[11] A. Pietsch, Operator ideals, North-Holland, 1980.

[12] M. Talagrand, Cotype and (q,1)-summing norms in Banach spaces, Invent. Math. 110 (1992), 545-556.

[Geraldo Botelho] Faculdade de Matemática, Universidade Federal de Uberlândia, 38.400-902 - Uberlândia, Brazil, e-mail: botelho@ufu.br.

[Daniel Pellegrino] Departamento de Matemática, Universidade Federal da Paraíba, 58.051-900 - João Pessoa, Brazil, e-mail: dmpellegrino@gmail.com.

[Pilar Rueda] Departamento de Análisis Matemático, Universidad de Valencia, 46.100 Burjasot - Valencia, Spain, e-mail: pilar.rueda@uv.es. 